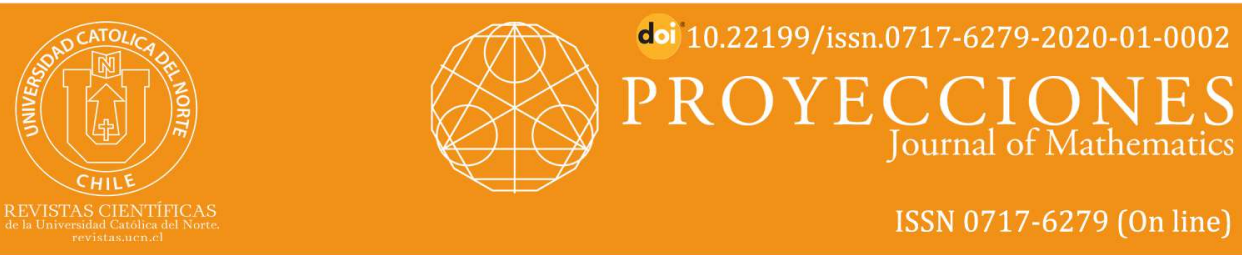

\title{
Nondifferentiable higher-order duality theorems for new type of dual model under generalized functions
}

Ramu Dubey ${ }^{1}$ @ orcid.org/0000-0001-8434-0907

Vishnu Narayan Mishra² orcid.org/0000-0002-2159-7710

${ }^{1}$ J. C. Bose University of Science and Technology, YMCA, Dept. of Mathematics, Faridabad, HR, India

rdubeyjiya@gmail.com

${ }^{2}$ Indira Gandhi National Tribal University, Dept. of Mathematics, Amarkantak, MP, India.

vishnunarayanmishra@gmail.com

Received: November 2018 | Accepted: December 2019

\section{Abstract:}

The motivation behind this article is to study a class of nondifferentiable multiobjective fractional programming problem in which each component of objective functions contains a term including the support function of a compact convex set. For a differentiable function, we consider a class of higher order pseudo quasi/ strictly pseudo quasi/weak strictly pseudo quasi- $(V, \rho, d)$-type-I convex functions. Under these the higher-order pseudo quasi/ strictly pseudo quasi/weak strictly pseudo quasi- $(V, \rho, d)$-type-I convexity assumptions, we prove the higher-order weak, higher-order strong and higher-order converse duality theorems related to efficient solution. establish Jensen-type and Hermite-Hadamard-type inequalities.

Keywords: Fractional programming; Multiobjective; Support function; Efficient solutions.

MSC (2010): 90C26; 90C30; 90C32; 90C46.

\section{Cite this article as (IEEE citation style)}

R. Dubey and V. N. Mishra, "Nondifferentiable higher-order

duality theorems for new type of dual model under generalized functions", Proyecciones (Antofagasta, On line), vol. 39, no. 1, pp. 15-29, Feb. 2020, doi: 10.22199/issn.0717-6279. 2020-01-0002. [Accessed dd-mm-yyyy]. 


\section{Introduction}

The fractional optimization problem with multiple objective functions have been the subject of intense investigations in the past few years, which have produced a number of optimality and duality results for these problems. Higher-order duality in non-linear programming has been studied in last few years by many researchers. In various numerical algorithms, higherorder duality is considered over first-order as it gives more closer bounds.

Higher-order duality in nonlinear programs have been studied by some researchers. Mangasarian [8] formulated a class of higher-order dual problems for the nonlinear programming problem "min $\{f(x): g(x) \geq 0\} "$ by introducing twice differentiable function $h: R^{n} \times R^{n} \rightarrow R$ and $k$ : $R^{n} \times R^{n} \rightarrow R^{m}$. The concept of higher-order convexity presented by Zhang [12] and derived duality results in multiobjective programming problem. Later on, Yang et al. [11] considered a unified higher-order dual model for nondifferentiable multiobjective programs and proved duality results under generalized assumptions. Suneja et al. [10] introduced a higher order $(F, \alpha, \sigma)$-type I functions and formulated higher order dual programs for a nondifferentiable multiobjective fractional programming problem.

Motivated by several concepts of generalized convexity, Gulati and Agarwal [4] gave the concept of second-order -V-type I functions for multiobjective programming problem which were recently extended to nondifferentiable case by Jayswal et al. [6]. Recently, Jayswal et al. [7] considerded higher-Order duality for multiobjective programming problems and discussed duality theorems under $(F, \alpha, \rho, d)-V$ - type-I functions. Several re searchers [[1], [2], [3], [9], [13]-[17]] have done their work in the related areas.

In this paper, we have generalized the definitions of higher-order pseudo quasi/ strictly pseudo quasi/weak strictly pseudo quasi- $(V, \rho, d)$-type-I functions for a nondifferentiable multiobjective higher-order fractional programming problem. We have formulated higher-order unified dual and established duality results under higher order pseudo quasi/ strictly pseudo quasi/weak strictly pseudo quasi- $(V, \rho, d)$-type-I assumptions. 


\section{Preliminaries and Definitions}

Throughout this paper, we use the index sets $K=\{1,2, \ldots, k\}$ and $M=$ $\{1,2, \ldots, m\}$.

Definition 2.1. Let $Q \subseteq R^{n}$ be a compact convex set. The support function of $Q$ is defined by

$$
s(y \mid Q)=\max \left\{y^{T} z: z \in Q\right\}
$$

Consider the following nondifferentiable multiobjective fractional programming problem:

$(\operatorname{MFP})$ Minimize $\Psi(x)=\left(\frac{\phi_{1}(x)+s\left(x \mid C_{1}\right)}{\psi_{1}(x)-s\left(x \mid E_{1}\right)}, \frac{\phi_{2}(x)+s\left(x \mid C_{2}\right)}{\psi_{2}(x)-s\left(x \mid E_{2}\right)}, \ldots, \frac{\phi_{k}(x)+s\left(x \mid C_{k}\right)}{\psi_{k}(x)-s\left(x \mid E_{k}\right)}\right)^{T}$

subject to $x \in Y^{0}=\left\{x \in Y: \pi_{j}(x)+s\left(x \mid D_{j}\right) \leq 0, j \in M\right\}$,

where $Y \subseteq R^{n}$ is an open set. The functions $\phi, \psi: Y \rightarrow R^{k}, \pi:$ $Y \rightarrow R^{m}$ are differentiable on $Y$ and $C_{i}, E_{i}, D_{j}$ are compact convex sets in $R^{n}$ for $i \in K$ and $j \in M$. Let $\phi_{i}(x)+s\left(x \mid C_{i}\right) \geq 0$ and $\psi_{i}(x)-$ $s\left(x \mid E_{i}\right)>0, i \in K$. Let $H=\left(H_{1}, H_{2}, \ldots, H_{k}\right): X \times R^{n} \rightarrow R^{k}$ and $K=\left(K_{1}, K_{2}, \ldots, K_{m}\right): X \times R^{n} \rightarrow R^{m}$ be differentiable functions, $d: X \times$ $X \rightarrow R, \quad z=\left(z_{1}, z_{2}, \ldots, z_{k}\right), \quad v=\left(v_{1}, v_{2}, \ldots, v_{k}\right)$ and $w=\left(w_{1}, w_{2}, \ldots, w_{m}\right)$, where $z_{i} \in C_{i}, v_{i} \in E_{i}$ and $w_{j} \in D_{j}$, for $i \in K$ and $j \in M$. Let $\rho=\left(\rho^{1}, \rho^{2}\right)$ such that $\rho^{1}=\left(\rho_{1}, \rho_{2}, \ldots, \rho_{k}\right) \in R^{k}, \rho^{2}=\left(\rho_{k+1}, \rho_{k+2}, \ldots, \rho_{k+m}\right) \in R^{m}$.

Definition 2.2. A point $u \in Y^{0}$ is efficient solution of (MFP) if $\exists$ no $x \in Y^{0}$ such that $\Psi(x) \leq \Psi(u)$.

Definition 2.3. $\forall i \in K, j \in M, \quad\left(\frac{\phi_{i}(.)+(.)^{T} z_{i}}{\psi_{i}(.)-(.)^{T} v_{i}}, \pi_{j}()+.(.)^{T} w_{j}\right)$ is higher order pseudo quasi $(V, \rho, d)$-type I at $u$ of (MFP) if, $\forall x \in Y^{0}$ and $p \in R^{n}$, such that 


$$
\begin{aligned}
& \frac{\phi_{i}(x)+x^{T} z_{i}}{\psi_{i}(x)-x^{T} v_{i}}<\frac{\phi_{i}(u)+u^{T} z_{i}}{\psi_{i}(u)-u^{T} v_{i}}+H_{i}(u, p)-p^{T} \nabla_{p} H_{i}(u, p) \\
& \Rightarrow \eta^{T}(x, u)\left\{\nabla\left(\frac{\phi_{i}(u)+u^{T} z_{i}}{\psi_{i}(u)-u^{T} v_{i}}\right)+\nabla_{p} H_{i}(u, p)\right\}+\rho_{i}^{1} d^{2}(x, u)<0
\end{aligned}
$$

and

$$
\begin{array}{r}
-\pi_{j}(u)-u^{T} w_{j} \leq K_{j}(u, p)-p^{T} \nabla_{p} K_{j}(u, p) \\
\Rightarrow \eta^{T}(x, u)\left\{\nabla \pi_{j}(u)+\nabla_{p} K_{j}(u, p)\right\}+\rho_{j}^{2} d^{2}(x, u) \leq 0 .
\end{array}
$$

Definition 2.4. $\forall i \in K, j \in M, \quad\left(\frac{\phi_{i}(.)+(.)^{T} z_{i}}{\psi_{i}(.)-(.)^{T} v_{i}}, \pi_{j}()+.(.)^{T} w_{j}\right)$ is higher order strictly pseudo quasi $(V, \rho, d)$-type I at $u$ of (MFP) if, $\forall x \in Y^{0}$ and $p \in R^{n}$, such that

$$
\begin{aligned}
& \frac{\phi_{i}(x)+x^{T} z_{i}}{\psi_{i}(x)-x^{T} v_{i}} \leq \frac{\phi_{i}(u)+u^{T} z_{i}}{\psi_{i}(u)-u^{T} v_{i}}+H_{i}(u, p)-p^{T} \nabla_{p} H_{i}(u, p) \\
& \Rightarrow \eta^{T}(x, u)\left\{\nabla\left(\frac{\phi_{i}(u)+u^{T} z_{i}}{\psi_{i}(u)-u^{T} v_{i}}\right)+\nabla_{p} H_{i}(u, p)\right\}+\rho_{i}^{1} d^{2}(x, u)<0
\end{aligned}
$$

and

$$
\begin{array}{r}
-\pi_{j}(u)-u^{T} w_{j} \leq K_{j}(u, p)-p^{T} \nabla_{p} K_{j}(u, p) \\
\Rightarrow \eta^{T}(x, u)\left\{\nabla \pi_{j}(u)+\nabla_{p} K_{j}(u, p)\right\}+\rho_{j}^{2} d^{2}(x, u) \leq 0 .
\end{array}
$$

Definition 2.5. $\forall i \in K, j \in M, \quad\left(\frac{\phi_{i}(.)+(.)^{T} z_{i}}{\psi_{i}(.)-(.)^{T} v_{i}}, \pi_{j}()+.(.)^{T} w_{j}\right)$ is higher order weak strictly pseudo quasi $(V, \rho, d)$-type I at $u$ of (MFP) if, $\forall x \in Y^{0}$ and $p \in R^{n}$, such that

$$
\frac{\phi_{i}(x)+x^{T} z_{i}}{\psi_{i}(x)-x^{T} v_{i}} \leq \frac{\phi_{i}(u)+u^{T} z_{i}}{\psi_{i}(u)-u^{T} v_{i}}+H_{i}(u, p)-p^{T} \nabla_{p} H_{i}(u, p)
$$




$$
\Rightarrow \eta^{T}(x, u)\left\{\nabla\left(\frac{\phi_{i}(u)+u^{T} z_{i}}{\psi_{i}(u)-u^{T} v_{i}}\right)+\nabla_{p} H_{i}(u, p)\right\}+\rho_{i}^{1} d^{2}(x, u)<0
$$

and

$$
\begin{array}{r}
-\pi_{j}(u)-u^{T} w_{j} \leq K_{j}(u, p)-p^{T} \nabla_{p} K_{j}(u, p) \\
\Rightarrow \eta^{T}(x, u)\left\{\nabla \pi_{j}(u)+\nabla_{p} K_{j}(u, p)\right\}+\rho_{j}^{2} d^{2}(x, u) \leq 0 .
\end{array}
$$

Definition 2.6. $\forall i \in K, j \in M,\left(\frac{\phi_{i}(.)+(.)^{T} z_{i}}{\psi_{i}(.)-(.)^{T} v_{i}}, \pi_{j}()+.(.)^{T} w_{j}\right)$ is higher order quasi strictly pseudo $(V, \rho, d)$-type I at $u$ of $(\mathrm{MFP})$ if, $\forall x \in Y^{0}$ and $p \in R^{n}$, such that

$$
\begin{aligned}
& \frac{\phi_{i}(x)+x^{T} z_{i}}{\psi_{i}(x)-x^{T} v_{i}} \leq \frac{\phi_{i}(u)+u^{T} z_{i}}{\psi_{i}(u)-u^{T} v_{i}}+H_{i}(u, p)-p^{T} \nabla_{p} H_{i}(u, p) \\
& \Rightarrow \eta^{T}(x, u)\left\{\nabla\left(\frac{\phi_{i}(u)+u^{T} z_{i}}{\psi_{i}(u)-u^{T} v_{i}}\right)+\nabla_{p} H_{i}(u, p)\right\}+\rho_{i}^{1} d^{2}(x, u) \leq 0
\end{aligned}
$$

and

$$
\begin{array}{r}
-\pi_{j}(u)-u^{T} w_{j} \leq K_{j}(u, p)-p^{T} \nabla_{p} K_{j}(u, p) \\
\Rightarrow \eta^{T}(x, u)\left\{\nabla \pi_{j}(u)+\nabla_{p} K_{j}(u, p)\right\}+\rho_{j}^{2} d^{2}(x, u)<0 .
\end{array}
$$

Theorem 2.1 (K-K-T-type necessary condition)[5]. Let $u$ be efficient solution of (MFP) at which the Kuhn-Tucker constraint qualification is satisfied on $X$. Then, $\exists 0<\bar{\lambda} \in R^{k}, 0 \leq \bar{y}_{j} \in R^{m}, \bar{z}_{i} \in R^{n}, \bar{v}_{i}, \bar{w}_{j} \in$ $R^{n}, i \in K, j \in M$ such that

$$
\begin{gathered}
\sum_{i=1}^{k} \bar{\lambda}_{i} \nabla\left(\frac{\phi_{i}(u)+u^{T} \bar{z}_{i}}{\psi_{i}(u)-u^{T} \bar{v}_{i}}\right)+\sum_{j=1}^{m} \bar{y}_{j} \nabla\left(\pi_{j}(u)+u^{T} \bar{w}_{j}\right)=0, \\
\sum_{j=1}^{m} \bar{y}_{j}\left(\pi_{j}(u)+u^{T} \bar{w}_{j}\right)=0,
\end{gathered}
$$




$$
\begin{gathered}
u^{T} \bar{z}_{i}=S\left(u \mid C_{i}\right), u^{T} \bar{v}_{i}=S\left(u \mid E_{i}\right), u^{T} \bar{w}_{j}=S\left(u \mid D_{j}\right), \\
\bar{z}_{i} \in C_{i}, \bar{v}_{i} \in D_{i}, \bar{w}_{j} \in E_{j}, i \in K, j \in M .
\end{gathered}
$$

In the following section, we consider the following mixed higher-order dual for (MFP) and derive duality theorems. The notation $\frac{\phi(.)+(.)^{T} z}{\psi(.)-(.)^{T} v}+$ $\mu_{J_{0}}^{T}\left(\pi_{j} J_{0}+(.)^{T} w J_{0}\right) e$ denotes the vector whose components are $\frac{\phi_{1}(.)+(.)^{T} z_{1}}{\psi_{1}(.)-(.)^{T} v_{1}}+$ $\sum_{j \in J_{0}} \mu_{j}\left(\pi_{j}+(.)^{T} w_{j}\right)$, $\frac{\phi_{2}(.)+(.)^{T} z_{2}}{\psi_{2}(.)-(.)^{T} v_{2}}+\sum_{j \in J_{0}} \mu_{j}\left(\pi_{j}+(.)^{T} w_{j}\right), \ldots, \frac{\phi_{k}(.)+(.)^{T} z_{k}}{\psi_{k}(.)-(.)^{T} v_{k}}+\sum_{j \in J_{0}} \mu_{j}\left(\pi_{j}+(.)^{T} w_{j}\right)$ and $\left\{\pi+(.)^{T} w\right\}_{J_{\beta}}^{\mu}$ denotes $r$-dimensional vector whose components are

$$
\sum_{j \in J_{1}}\left\{\pi_{j}+(.)^{T} w_{j}\right\}, \sum_{j \in J_{2}}\left\{\pi_{j}+(.)^{T} w_{j}\right\}, \ldots, \sum_{j \in J_{r}}\left\{\pi_{j}+(.)^{T} w_{j}\right\} .
$$

\section{Unified higher-order duality model:}

In this section, we formulate the following unified higher- order dual for (MFP) and establish duality theorems:

(HMDP) : Maximize $\left(\frac{\phi_{1}(y)+y^{T} z_{1}}{\psi_{1}(y)-y^{T} v_{1}}+H_{1}(y, p)-p^{T} \nabla_{p} H_{1}(y, p)+\sum_{j \in J_{0}} \mu_{j}\left\{\pi_{j}(y)+\right.\right.$ $\left.y^{T} w_{j}+K_{j}(y, p)-p^{T} \nabla_{p} K_{j}(y, p)\right\}, \ldots, \frac{\phi_{k}(y)+y^{T} z_{k}}{\psi_{k}(y)-y^{T} v_{k}}$ $\left.+H_{k}(y, p)-p^{T} \nabla_{p} H_{k}(y, p)+\sum_{j \in J_{0}} \mu_{j}\left\{\pi_{j}(y)+y^{T} w_{j}+K_{j}(y, p)-p^{T} \nabla_{p} K_{j}(y, p)\right\}\right)$ subject to $y \in Y$,

$$
\sum_{i=1}^{k} \lambda_{i}\left\{\nabla\left(\frac{\phi_{i}(y)+y^{T} z_{i}}{\psi_{i}(y)-y^{T} v_{i}}\right)+\nabla_{p} H_{i}(y, p)\right\}
$$




$$
\sum_{j \in J_{\beta}} \mu_{j}\left\{\pi_{j}(y)+y^{T} w_{j}+K_{j}(y, p)-p^{T} \nabla_{p} K_{j}(y, p)\right\} \geq 0, \beta=1, \ldots, r
$$

$$
\begin{gathered}
\lambda_{i} \geq 0, \quad \sum_{i=1}^{k} \lambda_{i}=1, \\
\mu_{j} \geq 0, z_{i} \in C_{i}, v_{i} \in E_{i}, w_{j} \in D_{j} \text { for } i \in K, j \in M,
\end{gathered}
$$

where $J_{\delta} \subseteq N, \delta=0,1, \ldots, r$ with $\bigcup_{\delta=0}^{r} J_{\delta}=N$ and $J_{\delta_{1}} \cap J_{\delta_{2}}$ if $\delta_{1} \neq \delta_{2}$. It may be noted that $J_{0}=N$ and $J_{\beta}=\phi(1 \leq \beta \leq r)$, we obtain Wolfe type dual. If $J_{0}=\phi, J_{1}=N$ and $J_{\beta}=\phi \quad(2 \leq \beta \leq r)$, then (HMDP) reduces to Mond-Weir Type dual.

Let $W^{0}$ be feasible solution of (HMDP).

Theorem 3.1 (Weak Duality Theorem). Let $x \in Y^{0}$ and $(y, \lambda, v, \mu, z, w, p) \in$ $W^{0}$. Let

(i) $\left(\frac{\phi_{i}(.)+(.)^{T} z_{i}}{\psi_{i}(.)-(.)^{T} v_{i}}+\mu_{J_{0}}^{T}\left(\pi_{j} J_{0}+(.)^{T} w_{j} J_{0}\right) e,\left\{\pi_{j}(.)+(.)^{T} w_{j}\right\}_{J_{\beta}}^{\mu}\right)$ be higherorder weak strictly pseudo quasi $(V, \rho, d)$-type I at $y$,

(ii) $\sum_{i=1}^{k} \lambda_{i} \rho_{i}^{1}+\sum_{j=1}^{r} \mu_{j} \rho_{j}^{2} \geq 0$.

Then, the following cannot hold

$\frac{\phi_{i}(x)+s\left(x \mid C_{i}\right)}{\psi_{i}(x)-s\left(x \mid E_{i}\right)} \leq \frac{\phi_{i}(y)+y^{T} z_{i}}{\psi_{i}(y)-y^{T} v_{i}}+H_{i}(y, p)-p^{T} \nabla_{p} H_{i}(y, p)$

$$
+\sum_{j \in J_{0}} \mu_{j}\left\{\pi_{j}(y)+y^{T} w_{j}+K_{j}(y, p)-p^{T} \nabla_{p} K_{j}(y, p)\right\}, \forall i \in K
$$


and

$$
\begin{aligned}
& \frac{\phi_{j}(x)+s\left(x \mid C_{j}\right)}{\psi_{j}(x)-s\left(x \mid E_{j}\right)}<\frac{\phi_{j}(y)+y^{T} z_{j}}{\psi_{j}(x)-y^{T} v_{j}}+H_{j}(y, p)-p^{T} \nabla_{p} H_{j}(y, p) \\
& \quad+\sum_{j \in J_{0}} \mu_{j}\left\{\pi_{j}(y)+y^{T} w_{j}+K_{j}(y, p)-p^{T} \nabla_{p} K_{j}(y, p)\right\}, \text { forsomej } \in K
\end{aligned}
$$

Proof: If possible, then suppose inequalities (3.5) and (3.6) hold. As $x^{T} z_{i} \leq s\left(x \mid C_{i}\right), x^{T} v_{i} \leq s\left(x \mid E_{i}\right), \forall i \in K$ and $\sum_{j \in J_{0}} \mu_{j}\left(\pi_{j}(x)+x^{T} w_{j}\right) \leq 0$, using the inequalities and the dual constraint (3.2), hypothesis $(i)$ gives

$$
\begin{array}{r}
\eta^{T}(x, y)\left\{\nabla\left(\frac{\phi_{i}(y)+y^{T} z_{i}}{\psi_{i}(y)-y^{T} v_{i}}\right)+\nabla_{p} H_{i}(y, p)+\sum_{j \in J_{0}} \mu_{j}\left\{\nabla \pi_{j}(y)+w_{j}+\nabla_{p} K_{j}(y, p)\right\} e\right\} \\
<-\rho^{1} d^{2}(x, y)
\end{array}
$$

and

$\eta^{T}(x, y) \sum_{j \in J_{\beta}} \mu_{j}\left\{\nabla \pi_{j}(y)+w_{j}+\nabla_{p} K_{j}(y, p)\right\}+\rho_{\beta}^{2} d^{2}(x, y) \leq 0, \beta=1, \ldots, r$.

Since $\lambda \geq 0, \lambda^{T} e=1$, it follows that

$$
\begin{aligned}
\eta^{T}(x, y) & \left(\sum_{i=1}^{k} \lambda_{i}\left\{\nabla\left(\frac{\phi_{i}(y)+y^{T} z_{i}}{\psi_{i}(y)-y^{T} v_{i}}\right)+\nabla_{p} H_{i}(y, p)\right\}\right. \\
+ & \left.\sum_{j \in J_{0}} \mu_{j}\left\{\nabla \pi_{j}(y)+w_{j}+\nabla_{p} K_{j}(y, p)\right\}\right)<-\sum_{i=1}^{k} \lambda_{i} \rho_{i}^{1} d^{2}(x, y)
\end{aligned}
$$

and

$$
\eta^{T}(x, y)\left(\sum_{j \in J_{\beta}} \mu_{j}\left\{\nabla \pi_{j}(y)+w_{j}+\nabla_{p} K_{j}(y, p)\right\}\right) \leq-\rho_{\beta}^{2} d^{2}(x, y), \beta=1, \ldots, r
$$


Above inequalities follows that

$$
\begin{gathered}
\eta^{T}(x, y)\left(\sum_{i=1}^{k} \lambda_{i}\left\{\nabla\left(\frac{\phi_{i}(y)+y^{T} z_{i}}{\psi_{i}(y)-y^{T} v_{i}}\right)+\nabla_{p} H_{i}(y, p)\right\}+\sum_{j=1}^{m} \mu_{j}\left\{\nabla \pi_{j}(y)+w_{j}\right.\right. \\
\left.\left.+\nabla_{p} K_{j}(y, p)\right\}\right)=\eta^{T}(x, y)\left(\sum_{i=1}^{k} \lambda_{i}\left\{\nabla\left(\frac{\phi_{i}(y)+y^{T} z_{i}}{\psi_{i}(y)-y^{T} v_{i}}\right)+\nabla_{p} H_{i}(y, p)\right\}\right. \\
+\sum_{j \in J_{0}} \mu_{j}\left\{\nabla \pi_{j}(y)+w_{j}+\nabla_{p} K_{j}(y, p)\right\}+\sum_{j \in J_{1}} \mu_{j}\left\{\nabla \pi_{j}(y)+w_{j}+\nabla_{p} K_{j}(y, p)\right\} \\
\left.+\ldots+\sum_{j \in J_{r}} \mu_{j}\left\{\nabla \pi_{j}(y)+w_{j}+\nabla_{p} K_{j}(y, p)\right\}\right) \\
\eta^{T}(x, y)\left(\sum_{i=1}^{k} \lambda_{i}\left\{\nabla\left(\frac{\phi_{i}(y)+y^{T} z_{i}}{\psi_{i}(y)-y^{T} v_{i}}\right)+\nabla_{p} H_{i}(y, p)\right\}+\sum_{j=1}^{m} \mu_{j}\left\{\nabla \pi_{j}(y)+w_{j}\right.\right. \\
\left.\left.+\nabla_{p} K_{j}(y, p)\right\}\right) \leq \eta^{T}(x, y)\left(\sum_{i=1}^{k} \lambda_{i}\left\{\nabla\left(\frac{\phi_{i}(y)+y^{T} z_{i}}{\psi_{i}(y)-y^{T} v_{i}}\right)+\nabla_{p} H_{i}(y, p)\right\}\right. \\
+\sum_{j \in J_{0}} \mu_{j}\left\{\nabla \pi_{j}(y)+w_{j}+\nabla_{p} K_{j}(y, p)\right\}+\sum_{j \in J_{1}} \mu_{j}\left\{\nabla \pi_{j}(y)+w_{j}\right. \\
\left.\left.+\nabla_{p} K_{j}(y, p)\right\}+\ldots+\sum_{j \in J_{r}} \mu_{j}\left\{\nabla \pi_{j}(y)+w_{j}+\nabla_{p} K_{j}(y, p)\right\}\right) \\
<-\left(\sum_{i=1}^{k} \lambda_{i} \rho_{i}^{1}+\sum_{j=1}^{r} \mu_{j} \rho_{j}^{2}\right) d^{2}(x, y) .
\end{gathered}
$$

Further, using hypothesis (ii), we have

$$
\begin{array}{r}
\eta^{T}(x, y)\left(\sum_{i=1}^{k} \lambda_{i}\left\{\nabla\left(\frac{\phi_{i}(y)+y^{T} z_{i}}{\psi_{i}(y)-y^{T} v_{i}}\right)+\nabla_{p} H_{i}(y, p)\right)\right\} \\
\left.+\sum_{j=1}^{m} \mu_{j}\left\{\nabla \pi_{j}(y)+w_{j}+\nabla_{p} K_{j}(y, p)\right\}\right)<0
\end{array}
$$

which contradicts (3.1). Hence, completes the proof. 
Theorem 3.2 (Weak Duality Theorem). Let $x \in Y^{0}$ and $(y, \lambda, v, \mu, z, w, p) \in$ $W^{0}$. Let

(i) $\left(\frac{\phi_{i}(.)+(.)^{T} z_{i}}{\psi_{i}(.)+(.)^{T} v_{i}}+\mu_{J_{0}}^{T}\left(\pi_{j} J_{0}+(.)^{T} w_{j} J_{0}\right) e,\left\{\pi_{j}(.)+(.)^{T} w_{j}\right\}_{J_{\beta}}^{\mu}\right)$ be higherorder pseudo quasi $(V, \rho, d)$-type I at $y$,

(ii) $\sum_{i=1}^{k} \lambda_{i} \rho_{i}^{1}+\sum_{j=1}^{r} \mu_{j} \rho_{j}^{2} \geq 0$.

Then, the following cannot hold

$$
\begin{aligned}
& \frac{\phi_{i}(x)+s\left(x \mid C_{i}\right)}{\psi_{i}(x)-s\left(x \mid E_{i}\right)} \leq \frac{\phi_{i}(y)+y^{T} z_{i}}{\psi_{i}(y)-y^{T} v_{i}}+H_{i}(y, p)-p^{T} \nabla_{p} H_{i}(y, p) \\
& \quad+\sum_{j \in J_{0}} \mu_{j}\left\{\pi_{j}(y)+y^{T} w_{j}+K_{j}(y, p)-p^{T} \nabla_{p} K_{j}(y, p)\right\}, \quad \forall i \in K
\end{aligned}
$$

and

$$
\begin{aligned}
& \frac{\phi_{j}(x)+s\left(x \mid C_{j}\right)}{\psi_{j}(x)-s\left(x \mid E_{j}\right)}<\frac{\phi_{j}(y)+y^{T} z_{j}}{\psi_{j}(x)-y^{T} v_{j}}+H_{j}(y, p)-p^{T} \nabla_{p} H_{j}(y, p) \\
& \quad+\sum_{j \in J_{0}} \mu_{j}\left\{\pi_{j}(y)+y^{T} w_{j}+K_{j}(y, p)-p^{T} \nabla_{p} K_{j}(y, p)\right\}, \text { for some } j \in K .
\end{aligned}
$$

Proof The proof follows on the lines of Theorem 3.1.

Theorem 3.3 (Strong Duality Theorem). If $\bar{u}$ is an efficient solution of (MFP) and let the Kuhn-Tucker constraint qualification be satisfied. Then, $\exists \bar{\lambda} \in R^{k}, \bar{y} \in R^{m}, \bar{z}_{i} \in R^{n}, \bar{v}_{i} \in R^{n}$ and $\bar{w}_{j} \in R^{n}, i \in K, j \in M$, such that $(\bar{u}, \bar{z}, \bar{v}, \bar{y}, \bar{\lambda}, \bar{w}, \bar{p}) \in W^{0}$ and the (MFP)and (HMDP) have equal values. Also, if

$$
H(\bar{u}, 0)=0, \nabla_{p} H(\bar{u}, 0)=0, K(\bar{u}, 0)=0, \nabla_{p} K(\bar{u}, 0)=0 .
$$


Furthermore, if the assumptions of Theorem 3.1 or 3.2 hold for $Y^{0}$ and $W^{0}$, then $(\bar{u}, \bar{z}, \bar{v}, \bar{y}, \bar{\lambda}, \bar{w}, \bar{p}=0)$ is an efficient solution of (HMDP).

Proof. Since $\bar{u}$ is an efficient solution for (MFP) and the Slaters constraint qualification is satisfied, from Theorem 2.1, there exist $0<\bar{\lambda} \in$ $R^{k}, 0 \leq \bar{y}_{j} \in R^{m}, \bar{z}_{i} \in R^{n}, \bar{v}_{i}, \bar{w}_{j} \in R^{n}, i \in K, j \in M$ such that

$$
\sum_{i=1}^{k} \bar{\lambda}_{i} \nabla\left(\frac{\phi_{i}(u)+u^{T} \bar{z}_{i}}{\psi_{i}(u)-u^{T} \bar{v}_{i}}\right)+\sum_{j=1}^{m} \bar{y}_{j} \nabla\left(\pi_{j}(u)+u^{T} \bar{w}_{j}\right)=0
$$

$$
\sum_{j=1}^{m} \bar{y}_{j}\left(\pi_{j}(u)+u^{T} \bar{w}_{j}\right)=0
$$

$$
\begin{gathered}
u^{T} \bar{z}_{i}=S\left(u \mid C_{i}\right), u^{T} \bar{v}_{i}=S\left(u \mid E_{i}\right), u^{T} \bar{w}_{j}=S\left(u \mid D_{j}\right), \\
\bar{z}_{i} \in C_{i}, \bar{v}_{i} \in D_{i}, \quad \bar{w}_{j} \in E_{j}, i \in K, j \in M .
\end{gathered}
$$

Using the assumption $H(\bar{u}, 0)=0, \nabla_{p} H(\bar{u}, 0)=0, K(\bar{u}, 0)=0, \nabla_{p} K(\bar{u}, 0)=$ 0 , we find that $(\bar{u}, \bar{z}, \bar{v}, \bar{y}, \bar{\lambda}, \bar{w}, \bar{p}=0) \in W^{0}$ and the two objective values are same. With the help of contradiction, we can prove efficiency results. Hence, the results.

Theorem 3.4 (Strict Converse Duality Theorem). Let $u \in Y^{0}$ and $(\bar{y}, \bar{\lambda}, \bar{\mu}, \bar{v}, \bar{z}, \bar{w}, \bar{p}) \in W^{0}$ such that

$$
\begin{aligned}
& \sum_{i=1}^{k} \overline{\lambda_{i}}\left\{\frac{\phi_{i}(u)+u^{T} \bar{z}_{i}}{\psi_{i}(u)-u^{T} \bar{v}_{i}}\right\} \leq \sum_{i=1}^{k} \overline{\lambda_{i}}\left\{\frac{\phi_{i}(\bar{y})+\bar{y}^{T} \bar{z}_{i}}{\psi_{i}(\bar{y})-\bar{y}^{T} \bar{v}_{i}}+\nabla \rho H_{i}(\bar{y}, \bar{p})-\bar{p}^{T} \nabla_{p} H_{i}(\bar{y}, \bar{p})\right. \\
+ & \sum_{j \in J_{0}} \bar{\mu}_{j}\left\{\pi_{j}(\bar{y})+\bar{y}^{T} \bar{w}_{j}+K_{j}(\bar{y}, \bar{p})-\bar{p}^{T} \nabla_{p} K_{j}(\bar{y}, \bar{p})\right\},
\end{aligned}
$$




$$
\begin{aligned}
& \rho_{i}^{1}+\sum_{j=1}^{r} \rho_{j}^{2} \geq 0, \forall i, j \\
& \left(\sum_{i=1}^{k} \overline{\lambda_{i}}\left\{\frac{\phi_{i}(.)+(.)^{T} \bar{z}_{i}}{\psi_{i}(.)-(.)^{T} \bar{v}_{i}}\right\}+\sum_{j \in J_{0}} \bar{\mu}_{j}\left\{\pi_{j}+(.)^{T} \bar{w}_{j}\right\},\left\{\pi_{j}(.)+(.)^{T} \bar{w}_{j}\right\}_{J_{\beta}}^{\bar{\mu}}\right)
\end{aligned}
$$

is higher order strictly pseudoquasi $(V, \rho, d)-$ typeIat $\bar{y}$.

Then, $u=\bar{y}$.

Proof. Suppose $u \neq \bar{y}$. The dual constraint (3.2) and the hypothesis (iii), for $\beta=1, \ldots, r$ yield

$$
\eta^{T}(u, \bar{y}) \sum_{j \in J_{\beta}} \bar{\mu}_{j}\left\{\nabla \pi_{j}(\bar{y})+\nabla \rho K_{j}(\bar{y}, p)+\bar{w}_{j}\right\} \leq-\rho_{\beta}^{2} d^{2}(u, \bar{y})
$$

By the dual constraint (3.1), we have

$$
\begin{array}{r}
\eta^{T}(u, \bar{y})\left(\sum_{i=1}^{k} \overline{\lambda_{i}}\left\{\nabla\left(\frac{\phi_{i}(u)+u^{T} \overline{z_{i}}}{\psi_{i}(u)-u^{T} \bar{v}_{i}}\right)+\nabla_{p} H_{i}(\bar{y}, \bar{p})\right\}\right. \\
\left.+\sum_{j=1}^{m} \overline{\mu_{j}}\left\{\nabla \pi_{j}(\bar{y})+\nabla_{p} K_{j}(\bar{y}, \bar{p})+\bar{w}_{j}\right\}\right)=0
\end{array}
$$

above inequalities with (3.13) give

$$
\begin{gathered}
\eta^{T}(u, \bar{y})\left(\sum_{i=1}^{k} \overline{\lambda_{i}}\left\{\nabla\left(\frac{\phi_{i}(u)+u^{T} \bar{z}_{i}}{\psi_{i}(u)-u^{T} \bar{v}_{i}}\right)+\nabla_{p} H_{i}(\bar{y}, \bar{p})\right\}+\sum_{j \in J_{0}} \overline{\mu_{j}}\left\{\nabla \pi_{j}(\bar{y})\right.\right. \\
\left.\left.+\nabla_{p} K_{j}(\bar{y}, \bar{p})+\bar{w}_{j}\right\}\right) \geq-\eta^{T}(u, \bar{y})\left(\sum_{j \in J_{1}} \overline{\mu_{j}}\left\{\nabla \pi_{j}(\bar{y})+\nabla_{p} K_{j}(\bar{y}, \bar{p})+\bar{w}_{j}\right\}\right) \\
-\ldots-\eta^{T}(u, \bar{y})\left(\sum_{j \in J_{r}} \overline{\mu_{j}}\left\{\nabla \pi_{j}(\bar{y})+\nabla_{p} K_{j}(\bar{y}, \bar{p})+\bar{w}_{j}\right\}\right)
\end{gathered}
$$

or 


$$
\begin{array}{r}
\eta^{T}(u, \bar{y})\left(\sum_{i=1}^{k} \overline{\lambda_{i}}\left\{\nabla\left(\frac{\phi_{i}(u)+u^{T} \bar{z}_{i}}{\psi_{i}(u)-u^{T} \bar{v}_{i}}\right)+\nabla_{p} H_{i}(\bar{y}, \bar{p})\right)\right\} \\
\left.+\sum_{j \in J_{0}} \overline{\mu_{j}}\left\{\nabla \pi_{j}(\bar{y})+\nabla_{p} K_{j}(\bar{y}, \bar{p})+\bar{w}_{j}\right\}\right) \geq \sum_{j=1}^{r} \rho_{j}^{2} d^{2}(u, \bar{y}),
\end{array}
$$

by hypothesis $(i i)$,

$$
\begin{array}{r}
\eta^{T}(u, \bar{y})\left(\sum_{i=1}^{k} \overline{\lambda_{i}}\left\{\nabla\left(\frac{\phi_{i}(u)+u^{T} \bar{z}_{i}}{\psi_{i}(u)-u^{T} \bar{v}_{i}}\right)+\nabla_{p} H_{i}(\bar{y}, \bar{p})\right\}\right. \\
\left.+\sum_{j \in J_{0}} \overline{\mu_{j}}\left\{\nabla \pi_{j}(\bar{y})+\nabla_{p} K_{j}(\bar{y}, \bar{p})+\bar{w}_{j}\right\}\right) \geq-\rho_{i}^{1} d^{2}(u, \bar{y}) .
\end{array}
$$

Therefore, hypothesis $(i i i)$ in view of $\sum_{j \in J_{0}} \mu_{j}\left\{\pi_{j}(u)+u^{T} \bar{w}_{j}\right\} \leq 0$ yields

$$
\begin{aligned}
\sum_{i=1}^{k} \overline{\lambda_{i}}\left\{\frac{\phi_{i}(u)+u^{T} \bar{z}_{i}}{\psi_{i}(u)-u^{T} \bar{v}_{i}}\right\}>\sum_{i=1}^{k} \overline{\lambda_{i}}\left\{\frac{\phi_{i}(\bar{y})+\bar{y}^{T} \bar{z}_{i}}{\psi_{i}(\bar{y})-\bar{y}^{T} \bar{v}_{i}}+\nabla p H_{i}(\bar{y}, \bar{p})-\bar{p}^{T} \nabla_{p} H_{i}(\bar{y}, \bar{p})\right\} \\
+\sum_{j \in J_{0}} \bar{\mu}_{j}\left\{\pi_{j}(\bar{y})+\bar{y}^{T} \bar{w}_{j}+K_{j}(\bar{y}, \bar{p})-\bar{p}^{T} \nabla_{p} K_{j}(\bar{y}, \bar{p})\right\}
\end{aligned}
$$

which contradicts hypothesis $(i)$. Hence, the result.

Theorem 3.5 (Strict Converse Duality Theorem). Let $u \in Y^{0}$ and $(\bar{y}, \bar{\lambda}, \bar{\mu}, \bar{v}, \bar{z}, \bar{w}, \bar{p}) \in W^{0}$ such that

$$
\begin{aligned}
& \text { (i) } \quad \sum_{i=1}^{k} \overline{\lambda_{i}}\left\{\frac{\phi_{i}(u)+u^{T} \bar{z}_{i}}{\psi_{i}(u)-u^{T} \bar{v}_{i}}\right\} \leq \sum_{i=1}^{k} \bar{\lambda}_{i}\left\{\frac{\phi_{i}(\bar{y})+\bar{y}^{T} \bar{z}_{i}}{\psi_{i}(\bar{y})-\bar{y}^{T} \bar{v}_{i}}+\nabla p H_{i}(\bar{y}, \bar{p})-\bar{p}^{T} \nabla_{p} H_{i}(\bar{y}, \bar{p})\right. \\
& +\sum_{j \in J_{0}} \bar{\mu}_{j}\left\{\pi_{j}(\bar{y})+\bar{y}^{T} \bar{w}_{j}+K_{j}(\bar{y}, \bar{p})-\bar{p}^{T} \nabla_{p} K_{j}(\bar{y}, \bar{p})\right\}, \\
& \text { (ii) } \quad \rho_{i}^{1}+\sum_{j=1}^{r} \rho_{j}^{2} \geq 0, \forall i, j,
\end{aligned}
$$


(iii) $\quad\left(\sum_{i=1}^{k} \overline{\lambda_{i}}\left\{\frac{\phi_{i}(.)+(.)^{T} \bar{z}_{i}}{\psi_{i}(.)-(.)^{T} \bar{v}_{i}}\right\}+\sum_{j \in J_{0}} \overline{\mu_{j}}\left\{\pi_{j}+(.)^{T} \bar{w}_{j}\right\},\left\{\pi_{j}(.)+(.)^{T} \bar{w}_{j}\right\}_{J_{\beta}}^{\bar{\mu}}\right)$

is higher order quasistrictly pseudo $(V, \rho, d)-$ typeIat $\bar{y}$.

Then, $u=\bar{y}$.

Proof The proof follows on the lines of theorem 3.4.

\section{References}

[1] R. Dubey and S. K. Gupta, "Duality for a nondifferentiable multiobjective higherorder symmetric fractional programming problems with cone constraints", Journal of nonlinear analysis and optimization, vol. 7, no, 1, pp. 1-15, 2016. [On line]. Available: https://bit.ly/2RRZaXg

[2] R. Dubey and V. N. Mishra, "Symmetric duality results for second-order nondifferentiable multiobjective programming problem", RAIRO - Operations research, vol. 53, no. 2, pp. 539-558, 2019, doi: 10.1051/ro/2019044.

[3] R. Dubey, V. N. Mishra, and P. Tomar, "Duality relations for second-order programming problem under $(G, \alpha f)$-bonvexity assumptions", Asian-European journal of mathematics, Art ID. 2050044, 2020, doi: 10.1142/S1793557120500448.

[4] T. R. Gulati and D. Agarwal, "Second-order duality in multiobjective programming involving $(F, \alpha, \rho, d)$-V-type I functions", Numerical functional analysis and optimization, vol. 28, no. 11-12, pp. 1263-1277, Oct. 2007, doi: 10.1080/01630560701749664.

[5] T. R. Gulati and Geeta, "Duality in nondifferentiable multiobjective fractional programming problem with generalized invexity", Journal of applied mathematics and computing, vol. 35, no. 1-2, pp. 103-118, Feb. 2009, doi: 10.1007/s12190009-0345-3.

[6] A. Jayswal, D. Kumar, and R. Kumar, "Second order duality for nondifferentiable multiobjective programming problem involving $(F, \alpha, \rho, d)$-V-type I functions", Optimization letters, vol. 4, no. 2, pp. 211-226, Nov. 2009, doi: 10.1007/s11590009-0159-0. 
[7] A. Jayswal, I. M. Stancu-Minasian, and D. Kumar, "Higher-order duality for multiobjective programming problems involving $(F, \alpha, \rho, d)$-V-type I functions", Journal of mathematical modelling and algorithms in operations research, vol. 13, no. 2, pp. 125-141, Apr. 2013, doi: 10.1007/s10852-013-9224-x.

[8] 0. Mangasarian, "Second- and higher-order duality in nonlinear programming", Journal of mathematical analysis and applications, vol. 51, no. 3, pp. 607-620, Sep. 1975, doi: 10.1016/0022-247X(75)90111-0.

[9] S. K. Mishra, K. K. Lai, and V. Singh, "Optimality and duality for minimax fractional programming with support function under $(C, \alpha, \rho, d)$-convexity", Journal of computational and applied mathematics, vol.274, pp.1-10,Jan. 2015, doi: 10.1016/j.cam.2014.06.025.

[10] S. K. Suneja, M. K. Srivastava, and M. Bhatia, "Higher order duality in multiobjective fractional programming with support functions", Journal of mathematical analysis and applications, vol. 347, no. 1,pp. 8-17, Nov.2008, doi: 10.1016/j.jmaa.2008.05.056.

[11] X. M. Yang, K. L. Teo, and X. Q. Yang, "Higher-order generalized convexity and duality in nondifferentiable multiobjective mathematical programming", Journal of mathematical analysis and applications, vol. 297, no. 1, pp. 48-55, Sep. 2004, doi: 10.1016/j.jmaa.2004.03.036.

[12] J. Zhang, "Higher order convexity and duality in multiobjective programming problems", in Progress in optimization. Contributions from australasia, vol. 30, A. Eberhard, R. Hill, D. Ralph, and B. M. Glover, Ed. Boston, MA: Springer, 1999, pp. 101117, doi: 10.1007/978-1-4613-3285-5_6.

[13] Vandana, R. Dubey, Deepmala, L. N. Mishra, and V. N. Mishra, "Duality relations for a class of a multiobjective fractional programming problem involving support functions", American journal of operations research, vol. 8, no. 4, pp. 294-311, Jul. 2018, doi: 10.4236/ajor.2018.84017.

[14] R. Dubey, Vandana, and V. N. Mishra, "Second order multiobjective symmetric programming problem and duality relations under (F, Gf)-convexity", Global journal of engineering science and researches, vol. 5, no. 8, pp. 187-199, 2018. [On line]. Available: https://bitly/30Ex5GM

[15] R. Dubey, L. N. Mishra, and L. M. S. Ruiz, "Nondifferentiable G-mond-weir type multiobjective symmetric fractional problem and their duality theorems under generalized assumptions", Symmetry, vol. 11, no. 11, Art. ID 1348, Jan. 2019, doi: $10.3390 /$ sym11111348.

[16] R. Dubey, L. N. Mishra, and C. Cesarano, "Multiobjective fractional symmetric duality in mathematical programming with $\left(C, G_{f}\right)$-invexity assumptions", Axioms, vol. 8, no. 3, Art. ID. 97, Aug. 2019, doi: 10.3390/axioms8030097.

[17] R. Dubey, L. N. Mishra, and R. Ali, "Special class of second-order non-differentiable symmetric duality problems with $(G, \alpha f)$-pseudobonvexity assumptions", Mathematics, vol. 7, no. 8, Art. ID 763, Aug. 2019, doi: 10.3390/math7080763. 